\title{
Pleomorphic liposarcoma arising on the cheek: a case report and review of the literature
}

\author{
Takahiro Suzuki*, Kazuhiro Murakami, Naoya Noguchi, Fumi Shoji, Sachiko Fukaya, \\ Yutaka Tateda, Muneharu Yamazaki, Miho Nakazumi, Teruyuki Sato, Nobuo Ohta
}

Division of Otolaryngology, Tohoku Medical and Pharmaceutical University, Sendai, Miyagi, Japan

Received: 16 June 2021

Accepted: 07 August 2021

\section{*Correspondence:}

Dr. Takahiro Suzuki,

E-mail: pilsaper@yahoo.co.jp

Copyright: ( ) the author(s), publisher and licensee Medip Academy. This is an open-access article distributed under the terms of the Creative Commons Attribution Non-Commercial License, which permits unrestricted non-commercial use, distribution, and reproduction in any medium, provided the original work is properly cited.

\begin{abstract}
Pleomorphic liposarcoma, which is the rarest of the four liposarcoma types according to the World Health Organization (WHO) classification of soft tissue tumors, is associated with a high rate of recurrence and poor prognosis. In the present report, we present the case of a 69-year-old male patient with pleomorphic liposarcoma arising in the right cheek. The mass was initially considered as benign such as schwannoma due to the absence of facial nerve palsy. However, the mass grew rapidly, requiring surgical removal with facial nerve preservation. Pathological and molecular examination led to the definitive diagnosis of pleomorphic liposarcoma. The postoperative adjuvant radiotherapy was added, and there were no signs of recurrence at four-year follow-up.
\end{abstract}

Keywords: H Pleomorphic liposarcoma, Cheek, Head and neck

\section{INTRODUCTION}

Liposarcomas are the most common soft tissue sarcomas, accounting for approximately $15 \%-20 \%$ of all soft tissue sarcomas). ${ }^{1}$ The majority of liposarcomas arise from the extremities and retroperitoneum), whereas only $4 \%$ of all liposarcomas originate in the head and neck region). ${ }^{2,3}$ Liposarcomas are classified into four types according to the World Health Organization (WHO) classification of soft tissue tumors: well-differentiated liposarcoma, which includes adipocytic, sclerosing, and inflammatory subtypes; dedifferentiated liposarcoma; myxoid liposarcoma; and pleomorphic liposarcoma (PLS). ${ }^{4} \mathrm{PLS}$ is the rarest liposarcoma type which can be distinguished from other high-grade sarcomas by the presence of pleomorphic lipoblasts. ${ }^{5}$ PLS located in head and neck region is exceedingly rare. Herein, we present a case of PLS arising on the right cheek and discuss the clinical course of the patient with review of the literature.

\section{CASE REPORT}

A 69-year-old male patient presented with one-month history of a painless mass on the right cheek. On physical examination, the mobile mass was hard with an elastic consistency and there were no signs of facial nerve palsy. Magnetic resonance imaging (MRI) revealed a wellcircumscribed and oval-shaped tumor, measuring $30 \times 24 \times 18 \mathrm{~mm}$, which was adjacent to the front edge of the right parotid gland. The mass, which was hypointense and homogeneously enhanced by gadolinium on T1-weighed images, was hyperintense on T2-weighed images. Fineneedle aspiration biopsy showed aggregations of spindle cells, suggesting a benign mesenchymal tumor. Considering the tumor location, schwannoma originating from the facial nerve was considered. The patient was followed due to the absence of facial nerve palsy. However, the tumor size increased rapidly by $1.3 \mathrm{~cm}$ in 3 months, measuring $43 \times 38 \times 29 \mathrm{~mm}$ (Figure 1). Therefore, a 
soft tissue tumor other than schwannoma was considered and tumor resection was planned.

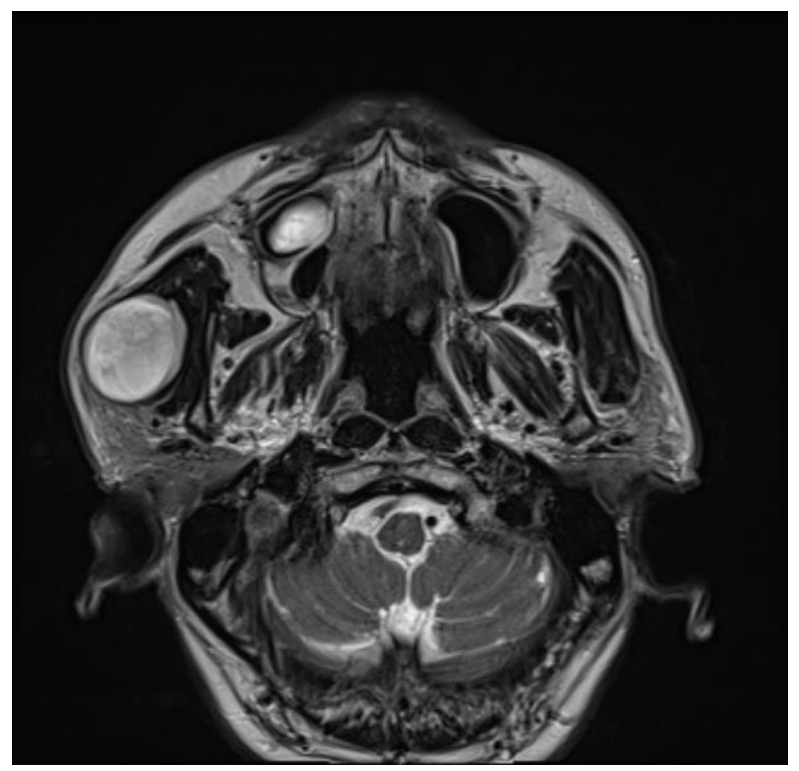

Figure 1: T2-weighed magnetic resonance image. Preoperative axial T2-weighted magnetic resonance image demonstrates a well-circumscribed and ovalshaped tumor located in front of the right parotid gland, measuring $43 \times 38 \times 29 \mathrm{~mm}$.

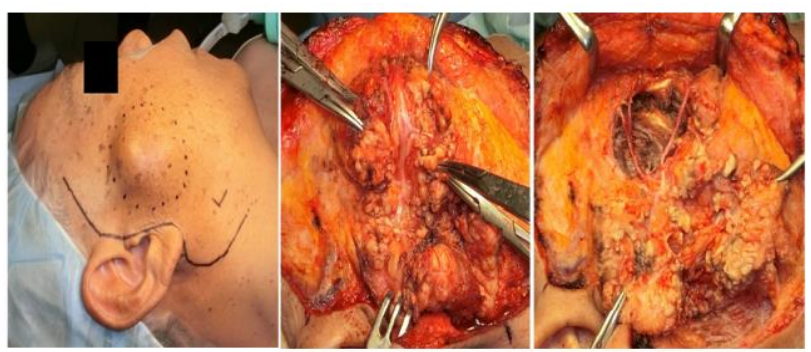

Figure 2: Surgical findings. (A) S-shaped skin incision extended to the sideburns, (B) the tumor is localized beneath the buccal branch of facial nerve and (C) surgical field after tumor resection. White arrowhead, buccal branch; black arrow heads, zygomatic branches; T, tumor.

An S-shaped skin incision in the right side was performed to approach the lesion (Figure 2a), and the facial nerve was identified. The tumor was located beneath the buccal branch (Figure $2 b$ ) and on the surface of the zygomatic branches of facial nerve. These branches were preserved as macroscopic tumor infiltration was not observed, although some of the branches were adjacent to the tumor. The tumor was resected together with part of the masseter muscle (Figure 2c). The excised specimen was a solid, milky white mass infiltrating the surrounding masseter muscle (Figure 3). Intraoperative pathological examination of the frozen section revealed a mesenchymal tumor, but the presence of malignancy could not be confirmed. The patient, who was discharged on postoperative day seven, experienced postoperative facial nerve weakness in the zygomatic and buccal areas but recovered two months after the surgery.

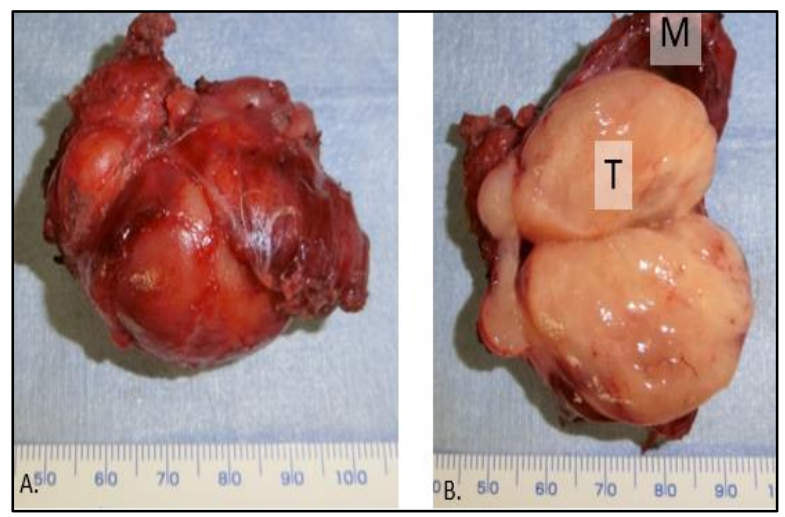

Figure 3: Surgical specimen. (A) Gross appearance of the tumor, (B) cut tumor surface with milky white color is partially infiltrating the masseter muscle. $T$, tumor; M, masseter muscle.

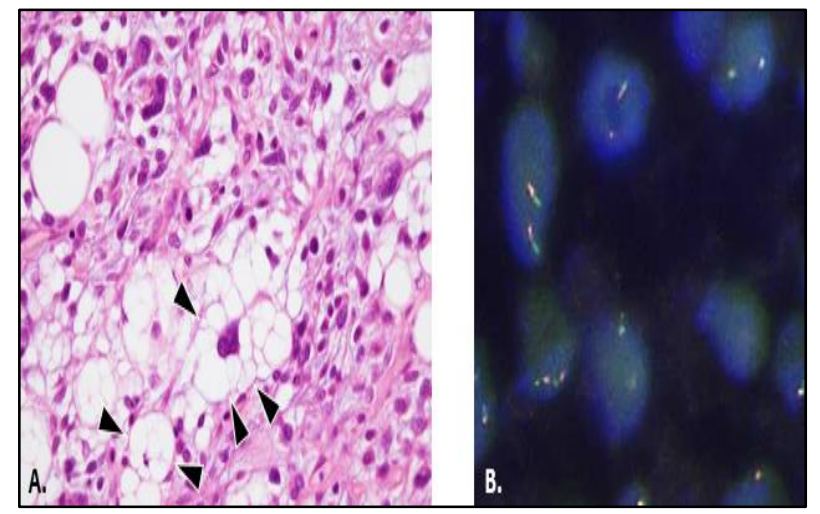

Figure 4: Histologic findings and fluorescent in situ hybridization. (A) Histologic section showing plenty of lipoblasts (black arrowhead) with microvacuolated cytoplasm and scalloped nucleus (hematoxylin/eosin staining, original magnification 200x), (B) the DDIT3 break-apart probe comprises one green and one red probe, which flank the region of DDIT3. Presence of split green and red probes indicates the absence of a rearrangement in DDIT3.

Histopathological examination of the resected tumor revealed spindle cells with marked nuclear pleomorphism and abundant lipoblasts with microvacuolated cytoplasm and scalloped nuclei; these findings suggested PLS (Figure 4a). Immunohistochemical analysis revealed focal areas positive for CD34, CD10, and S-100, and the Ki-67 index was $40 \%-50 \%$. The tumor cells did not express nuclear MDM2 or CDK4. Fluorescence in situ hybridization (FISH) analysis did not reveal MDM2 amplification, leading to the exclusion of well-differentiated liposarcoma and dedifferentiated liposarcoma. FISH analysis also failed to show DDIT3 gene rearrangement (Figure 4b), leading to the exclusion of myxoid liposarcoma. Therefore, the definitive diagnosis was PLS. 
The postexcision margin was not tumor-free, and the patient was recommended postoperative radiotherapy to reduce the risk of local recurrence. He was treated with a total dose of $60 \mathrm{~Gy}$. At last visit after four years of followup with imaging studies, there were no signs of tumor relapse or metastasis.

\section{DISCUSSION}

The histologic classification of liposarcomas is important for prognostic prediction and determination of treatment. well-differentiated liposarcoma and dedifferentiated liposarcoma are the most common histologic liposarcoma types, accounting for $40 \%-50 \%$ of all liposarcomas. ${ }^{6}$ Well-differentiated liposarcoma is a non-metastasizing low-grade tumor with tendency for local recurrence, whereas dedifferentiated liposarcoma represents the progression of well-differentiated liposarcoma from an indolent, sometimes locally aggressive mass to a more rapidly growing tumor with metastatic potential. ${ }^{7,8}$ Myxoid liposarcoma, which accounts for approximately $30 \%$ of all liposarcomas, includes the round-cell subtype characterized by high-grade malignancy. ${ }^{6}$ Aggressiveness in myxoid liposarcoma is associated with the percentage of round-cell component, leading to an unfavorable prognosis. $^{9}$ PLS, which is a high-grade malignant liposarcoma with high recurrence rate and poor prognosis, is very rare and accounts for only $5 \%-10 \%$ of all liposarcoma cases. ${ }^{10}$

The definite diagnosis of PLS depends on pathological examination, which reveals highly metatypical cells with granular and/or foamy small cytoplasmic vacuoles, mononuclear/polynuclear giant cells with strongly stained nucleoli, and pathological karyokinesis accompanied with scattered, highly specific spindle cells. ${ }^{11,12}$ Molecular examination is also useful in determining specific liposarcoma types. Well-differentiated liposarcoma and dedifferentiated liposarcoma are nearly universally associated with the amplification of $12 \mathrm{q}$, which includes the proto-oncogenes MDM2, CDK4, and HMGA27), whereas MDM2 amplification is not a consistent feature of PLS. ${ }^{13-15}$ Myxoid liposarcoma is almost always associated with chromosomal translocations, most commonly $\mathrm{t}(12 ; 16)$ (q13;p11), in more than $90 \%$ of cases. This translocation leads to the fusion of DDIT3 and FUS, resulting in the formation of FUS-DDIT3 fusion protein. ${ }^{15}$ The genetic features of PLS are nonspecific, and our understanding remains limited. PLS is a complexkaryotype sarcoma characterized by complex focal copy number changes throughout the genome, and both TP53 and RB1 are frequently lost in PLS. ${ }^{6,16}$ Cho et al reported a critical diagnosis revision rate of $3.5 \%$ (5/142) after their review of lipomatous lesions using FISH analysis of MDM2 and DDIT3, reflecting the value of molecular assessment for accurate subtyping of liposarcomas. ${ }^{14}$ In the present case, the diagnosis of PLS was based on microscopic examination, which was confirmed by FISH analysis that showed the absence of MDM2 amplification and DDIT3 rearrangement.
In the present case, preoperative definitive diagnosis, including the presence of malignancy, was not possible based on fine-needle aspiration biopsy and MRI. Open biopsy was avoided considering the possibility that the lesion was a benign parotid tumor, such as pleomorphic adenoma. However, the rapid tumor growth led to the decision of resection, with planning for facial nerve reconstruction in case of concomitant resection of the facial nerve. The tumor resection was possible with facial nerve preservation, although some of the branches were close to the tumor. The principal treatment of liposarcoma is complete excision, which should be as wide and meticulous as possible, extending at least $2 \mathrm{~cm}$ from the palpable tumor margin. ${ }^{17-19}$ Therefore, the safety margin of the excision was inadequate due to facial nerve preservation. Given that incomplete excision is associated with increased risk of local recurrence, postoperative radiotherapy is increasingly considered for such cases. ${ }^{3}$ Several studies reported that the rate of local recurrence was reduced in patients who received postoperative radiotherapy. Spittle et al reported that only $20 \%$ of liposarcoma recurred locally in patients who received radiotherapy compared with the recurrence rate of $73 \%$ in those who did not receive radiotherapy. ${ }^{20}$ Eeles et al reported that the addition of radiotherapy to surgery for the treatment of head and neck sarcomas significantly decreased the rate of local recurrence from $60 \%$ to $40 \%$, although a significant impact on overall survival was not observed..$^{21}$ Radiation should be considered especially for patients with high-grade tumors, positive margins, large tumors, and complex anatomic subsites. ${ }^{22,23}$ Although its curative effect in PLS remains controversial, chemotherapy should be considered in patients with distant metastases. Doxorubicin and ifosfamide are used as adjuvant chemotherapy in metastatic PLS, and PLS may also respond to gemcitabine-based therapies. ${ }^{7}$

PLS typically arises in extremities and retroperitoneum. ${ }^{2}$ Approximate local recurrence and metastatic rates are $30 \%-50 \%$ and $50 \%$, respectively and tumor-associated mortality occurs in up to $50 \%$ of patients. ${ }^{5,11,24}$ Several studies reported PLS in the head and neck region. Wang et al reported the case of a patient with LPS originating from the posterior occipital region, which was surgically excised; the tumor recurred five times within five years. ${ }^{12}$ Davis et al reported 11 cases, including 1, 7, 2, and 1 case with PLS in scalp/face, neck/parotid, upper aerodigestive tract, and skull base/orbit, respectively; in that cohort, treatments were surgery alone, surgery and radiotherapy with or without chemotherapy, radiotherapy alone, and radiotherapy plus chemotherapy in $6,3,1$, and 1 case, respectively. ${ }^{23}$ The authors reported local recurrence and distant metastasis in $5(45.5 \%)$ and $3(27.3 \%)$ cases, respectively. Gritlis et al reported 4 PLS cases, including 2, 1 , and 1 case involving gums, cheek, and hard palate, respectively. ${ }^{19}$ The treatments included surgery alone, surgery plus radiotherapy, and radiotherapy alone in 2,1 , and 1 case, respectively, and all patients recurred. Agarwal et al reported two cases, including the cheek and postauricular region in one case each; both patients were 
treated by surgery followed by adjuvant radiotherapy and did not have evidence of recurrence at one year followup. ${ }^{22}$ These case reports suggest that PLS can arise anywhere in the head and neck region, with no clear tendency to originate from a specific location. The mainstay of treatment for PLS is surgical excision, and adjuvant radiotherapy is often used, as reported in studies to date. The recurrence rate is high, suggesting severe aggressiveness of PLS in the head and neck region similar to that observed in PLS arising from other sites including the extremities and retroperitoneum.

\section{CONCLUSION}

We herein presented a case of PLS arising on the right cheek. The patient recognized the mass and sought medical attention early. Preoperative diagnosis based on fine-needle aspiration biopsy and MRI was challenging. The lesion was surgically removed, and the patient received adjuvant radiotherapy after the definitive diagnosis based on histopathological and molecular examination, with no recurrence at four years after surgery. PLS of the head and neck is extremely rare; therefore, literature on this disease is limited and standard treatment for PLS remains controversial. Studies with larger cohorts are necessary to develop therapeutic strategies for PLS.

Funding: No funding sources

Conflict of interest: None declared

Ethical approval: Not required

\section{REFERENCES}

1. Ducimetière $F$, Lurkin $A$, Ranchère-Vince $D$, Decouvelaere AV, Péoc'h M, Istier L et al. Incidence of sarcoma histotypes and molecular subtypes in a prospective epidemiological study with central pathology review and molecular testing. PLoS One. 2011;6:e20294.

2. Reitan JB, Kaalhus O, Brennhovd IO, Sager EM, Stenwig AE, Talle $\mathrm{K}$ et al. Prognostic factors in liposarcoma. Cancer. 1985;55:2482-90.

3. Golledge J, Fisher C, Rhys-Evans PH. Head and neck liposarcoma. Cancer. 1995;76:1051-8.

4. Jo VY, Fletcher CD. WHO classification of soft tissue tumours: an update based on the 2013 (4th) edition. Pathology. 2014;46:95-104.

5. Downes KA, Goldblum JR, Montgomery EA, Fisher C. Pleomorphic liposarcoma: a clinicopathologic analysis of 19 cases. Mod Pathol. 2001;14:179-84.

6. Lee ATJ, Thway K, Huang PH, Jones RL. Clinical and molecular spectrum of liposarcoma. J Clin Oncol. 2018;36:151-9.

7. Crago AM, Dickson MA. Liposarcoma: multimodality management and future targeted therapies. Surg Oncol Clin N Am. 2016;25:761-73.

8. Dalal KM, Kattan MW, Antonescu CR, Brennan MF, Singer S. Subtype specific prognostic nomogram for patients with primary liposarcoma of the retroperitoneum, extremity, or trunk. Ann Surg. 2006;244:381-91.

9. Kilpatrick SE, Doyon J, Choong PFM, Sim FH, Nascimento AG. The clinicopathologic spectrum of myxoid and round cell liposarcoma: a study of 95 cases. Cancer. 1996;77:1450-8.

10. Peterson JJ, Kransdorf MJ, Bancroft LW, O'Connor MI. Malignant fatty tumors: classification, clinical course, imaging appearance and treatment. Skeletal Radiol. 2003;32:493-503.

11. Hornick JL, Bosenberg MW, Mentzel T, McMenamin ME, Oliveira AM, Fletcher CDM. Pleomorphic liposarcoma: clinicopathologic analysis of 57 cases. Am J Surg Pathol. 2004;28:1257-67.

12. Wang L, Luo R, Xiong Z, Xu J, Fang D. Pleomorphic liposarcoma: an analysis of 6 case reports and literature review. Medicine (Baltimore). 2018;97:e9986.

13. Sandberg AA. Updates on the cytogenetics and molecular genetics of bone and soft tissue tumors: liposarcoma. Cancer Genet Cytogenet. 2004;155:124.

14. Cho J, Lee SE, Choi Y-L. Diagnostic Value of MDM2 and DDIT3 Fluorescence in situ hybridization in liposarcoma classification: a singleinstitution experience. Korean J Pathol. 2012;46:11522.

15. Keung EZ, Somaiah N. Overview of liposarcomas and their genomic landscape. J Transl Genet Genom. 2019;3:8.

16. Guillou L, Aurias A. Soft tissue sarcomas with complex genomic profiles. Virchows Arch 2010;456:201-17.

17. Ohta N, Watanabe T, Abe Y, Onoe Y, Ishida A, Aoyagi M. Dedifferentiated posterior cervical liposarcoma with carcinoid: report of a case. Auris Nasus Larynx. 2011;38:421-5.

18. Mouret P. Liposarcoma of the hypopharynx. A case report and review of the literature. Rev Laryngol Otol Rhinol. 1999;120:39-43.

19. Gritli S, Khamasi K, Lachkhem A, Touati S, Chorfa A, Ben MT, et al. Head and neck liposarcoma: a 32 year experience. Auris Nasus Larynx. 2010;37:34751.

20. Spittle MF, Newton A, MacKenzie DH. Liposarcoma: a review of 60 cases. Br J Cancer. 1970;24:696-704.

21. Eeles RA, Fisher C, A'Hem RP, Robinson M, RhysEvans P, Henk JM, et al. Head and neck sarcomas: prognostic factors and implications for treatment. $\mathrm{Br}$ J Cancer. 1993;68:201-7.

22. Agarwal J, Kadakia S, Agaimy A, Ogadzanov A, Khorsandi A, Chai RL. Pleomorphic liposarcoma of the head and neck: presentation of two cases and literature review. Am J Otolaryngol. 2017;38:505-7.

23. Davis EC, Ballo MT, Luna MA, Roberts DB, Nong $\mathrm{X}$. Liposarcoma of the head and neck: the University of Texas MD Anderson Cancer Center experience. Head Neck. 2009;31:28-36. 
24. Gebhard S, Coindre JM, Michels JJ, Terrier P, Bertrand G, Trassard M, et al. Pleomorphic liposarcoma: clinicopathologic, immunohistochemical, and follow-up analysis of 63 cases: a study from the French Federation of Cancer Centers Sarcoma Group. Am J Surg Pathol. 2002;26:601-16.
Cite this article as: Suzuki T, Murakami K, Noguchi N, Shoji F, Fukaya S, Tateda Y et al. Pleomorphic liposarcoma arising on the cheek: a case report and review of the literature. Int J Otorhinolaryngol Head Neck Surg 2021;7:1526-30. 